\title{
THE BAER-SPECKER GROUP
}

\section{Eoin Coleman ${ }^{1}$}

The Baer-Specker group, $\mathbf{P}$, is the group of functions from the natural numbers $\mathbf{N}$ into the integers $\mathbf{Z}$. While $\mathbf{P}$ is very easy to define, it is the source of a wealth of problems, some of which have only recently been solved. The aim of this paper is to present an introductory account of old and new results about $\mathbf{P}$, and to explore some of the connections of this group with other areas of mathematics, in particular with the real numbers, infinitary logic, and combinatorial set theory.

\section{Introduction}

The Baer-Specker group $\mathbf{P}$ is an infinite abelian group under the addition

$$
(f+g)(n)=f(n)+g(n)
$$

for $n \in \mathbf{N}$. $\mathbf{P}$ contains the subgroup $\mathbf{S}$, the direct sum of countably many copies of $\mathbf{Z}$. In the literature, $\mathbf{P}$ is also denoted $\mathbf{Z}^{\mathbf{N}}$, or $\mathbf{Z}^{\omega}$. Since all the groups considered in this paper are abelian, it will save space to adopt the convention that the term group is short for abelian group. The textbooks [20] and [22] are good references for infinite abelian group theory. We use the symbols $\omega, \omega_{1}$, and $2^{\omega}$ to denote the cardinal numbers of the natural numbers $\mathbf{N}$, the first uncountable cardinal, and the cardinal number of the real numbers $\mathbf{R}$, respectively. For example, $\mathbf{P}$ has cardinality $2^{\omega}$, and $\mathbf{S}$ has cardinality $\omega$.

\footnotetext{
${ }^{1}$ I am very grateful to the organizers of the Irish Mathematical Society Conference in September 1997 for the opportunity to read a shorter version of this paper to the participants.
} 
There are four sections in the paper. The first resumes some basic material on $\mathbf{P}$; the second looks at a recently discovered connection relating slender subgroups of $\mathbf{P}$ to certain cardinal invariants of the real numbers. In the third section, some logical aspects of $\mathbf{P}$ are explored. The final section is about the complexity of the lattice of subgroups of $\mathbf{P}$. Since the material covered in the paper is diverse and scattered across different domains, I have not given many proofs, hoping that the bibliography will enable the reader to follow themes in more depth.

\section{Basics}

The structure of infinite free groups is relatively clear (see, for example, [22]): for each infinite cardinal $\kappa$, there is exactly one free group of cardinality $\kappa$ on $\kappa$ generators (up to isomorphism). So an obvious initial question in the study of $\mathbf{P}$ is to determine how it stands in relation to freeness: is $\mathbf{P}$ free?

Definition A group $G$ is free if $G$ is (isomorphic to) a direct sum of copies of $\mathbf{Z}$.

For example, $\mathbf{P}$ has a free subgroup $\mathbf{S}$.

Theorem 1.1 (Baer [1]) The group $\mathbf{P}$ is not free.

We shall deduce this theorem from a stronger assertion below involving the notion of $\kappa$-freeness.

Definition Suppose that $\kappa$ is an infinite cardinal. A group $G$ is $\kappa$-free if every subgroup $H \leq G$ having less than $\kappa$ elements is free.

Every free group is $\kappa$-free for every cardinal $\kappa$, since subgroups of free groups are free; if $\lambda<\kappa$, then $\kappa$-freeness implies $\lambda$ freeness. Questions about whether $\lambda$-freeness implies $\kappa$-freeness for $\lambda<\kappa$ are highly non-trivial and have stimulated one of the most important research orientations in infinite abelian group theory, leading for example to Shelah's singular compactness theorem $[7,25,20]$ and independence results in set theory [30]. Since in general $\kappa$-freeness is weaker than freeness, we can refine the initial question about the non-freeness of $\mathbf{P}$ and ask whether there are any cardinals $\kappa$ for which $\mathbf{P}$ is $\kappa$-free. Recall that $\omega_{2}$ is the second uncountable cardinal, the cardinal successor of $\omega_{1}$. 
Proposition 1.2 The group $\mathbf{P}$ is not $\omega_{2}$-free.

Proof: We need to find a non-free subgroup of $\mathbf{P}$ which has cardinality $\omega_{1}$. Let $p$ be a fixed prime number, and take a pure subgroup $H$ of cardinality $\omega_{1}$ containing $\mathbf{S}$ and such that every element $\left(n_{1}, n_{2}, \ldots\right)$ of $H$ has the property that the tail is divisible by arbitrarily high powers of $p:(\forall m)(\exists r)(\forall k>r)\left(p^{m} \mid n_{k}\right) .^{2}$ Then the quotient group $H / p H$ is a vector space over $\mathbf{F}_{p}$, the finite field of $p$ elements. It follows that $H$ is not free, for if $H$ were free, then $H / p H$ must have dimension (and hence cardinality) $\omega_{1}$; but every coset of $H / p H$ contains an element of $\mathbf{S}$, and hence $H / p H$ has cardinality at most $|\mathbf{S}|=\omega-$ a contradiction. So $H$ is not free.

We can use Proposition 1.2 to improve exercise 19.7 of [22]:

Corollary For every uncountable cardinal $\kappa$, there exists a nonfree $\omega_{1}$-free group of cardinality $\kappa$.

Proof: For example, the group $\oplus_{\alpha<\kappa} H$, the direct sum of $\kappa$ copies of the group $H$ in the proof of Proposition 1.2, will work.

Proposition 1.2 implies Baer's theorem, since $H$ is a nonfree subgroup of $\mathbf{P}$. However, it leaves open the question whether countable subgroups of $\mathbf{P}$ are free, i.e. whether $\mathbf{P}$ is $\omega_{1}$-free.

Theorem 1.3 (Specker [42]) The group $\mathbf{P}$ is $\omega_{1}$-free.

Proof: This is a well-known result and a full proof is given in the standard reference textbooks [20, 22]. It rests on Pontryagin's Criterion: a countable group is free if and only if every finite rank subgroup is free. We shall give a proof of this criterion using logic in section three. The proof of Theorem 1.3 proceeds as follows: every finite rank subgroup of $\mathbf{P}$ is embedded in a finitely generated torsion-free direct summand of $\mathbf{P}$, and hence is free; so if $G \leq \mathbf{P}$ is countable, then every finite rank subgroup of $G$ is free; now apply Pontryagin's Criterion.

To close this section, let us introduce another type of "local" freeness which has been intensively studied: a group $G$ is almost free if $G$ is $|G|$-free $(|G|$ is the number of elements of $G$ ), i.e. every

\footnotetext{
2 Or: let $H$ be an elementary submodel of the $p$-adic closure of $\mathbf{S}$ in $\mathbf{P}$ of cardinality $\omega_{1}$ containing $\mathbf{S}$. $H$ exists by the Downward LoewenheimSkolem Theorem of first-order logic.
} 
subgroup of $G$ of smaller cardinality than $G$ is free. Is $\mathbf{P}$ almost free?

Corollary 1.4 P is almost free if and only if the Continuum Hypothesis (CH: $\left.2^{\omega}=\omega_{1}\right)$ holds.

If the Continuum Hypothesis is true, then $\mathbf{P}$ is almost free if $\mathbf{P}$ is $\omega_{1}$-free, which is true by Theorem 1.3; if the Continuum Hypothesis is false, then the subgroup $H$ in Proposition 1.2 is a non-free subgroup of cardinality $\omega_{1}<2^{\omega}=|\mathbf{P}|$ and so $\mathbf{P}$ is not almost free. Thus one cannot decide whether $\mathbf{P}$ is almost free or not on the basis of ordinary set theory (ZFC).

One trend in the study of $\kappa$-freeness has been to try to find equivalences between the algebraic and set-theoretic definitions. In light of Corollary 1.4, it might be interesting to know whether there are algebraic properties $\varphi$ and $\psi$ such that:

(1) $\mathbf{P}$ has almost $\varphi$ iff the weak Continuum Hypothesis $(\mathbf{w} \mathbf{C H}$ : $\left.2^{\omega}<2^{\omega_{1}}\right)$ holds;

(2) $\mathbf{P}$ has almost $\psi$ iff Diamond holds.

Diamond $\diamond$ is a stronger form of the Continuum Hypothesis $\mathbf{C H}$. One way to state $\mathbf{C H}$ is as a list of guesses $A_{\alpha}$ for the subsets of $\mathbf{N}$ :

$$
\begin{aligned}
& \left(\exists\left\{A_{\alpha} \subseteq \alpha: \alpha<\omega_{1}\right\}\right. \text { such that } \\
& \left.(\forall X \subseteq \mathbf{N})\left(\left\{\alpha: X=A_{\alpha}\right\} \text { is a stationary subset of } \omega_{1}\right)\right) .
\end{aligned}
$$

A stationary subset of $\omega_{1}$ is large: it intersects every closed unbounded subset of $\omega_{1}$ (in the order topology) non-trivially. In other words, the Continuum Hypothesis says that there is a list of length $\omega_{1}$ which predicts correctly every subset of natural numbers a large number of times. What about subsets of $\omega_{1}$ ? One cannot hope for a list of length $\omega_{1}$ which would predict correctly every subset of $\omega_{1}$, since there are $2^{\omega_{1}}$ subsets of $\omega_{1}$ and $2^{\omega_{1}}>\omega_{1}$. But perhaps one might be able to predict correctly just the initial segments of subsets of $\omega_{1}$. Diamond asserts that there is a list of $\omega_{1}$ guesses $A_{\alpha}$ for the initial segments of subsets of $\omega_{1}$ and these guesses are correct on a large subset of $\omega_{1}$. Formally, Diamond 
states:

$$
\begin{aligned}
& \left(\exists\left\{A_{\alpha} \subseteq \alpha: \alpha<\omega_{1}\right\}\right. \text { such that } \\
& \left.\left(\forall X \subseteq \omega_{1}\right)\left(\left\{\alpha: X \cap \alpha=A_{\alpha}\right\} \text { is a stationary subset of } \omega_{1}\right)\right) .
\end{aligned}
$$

A more detailed explanation of this type of prediction principle can be found in the standard textbooks on set theory [26] and [29].

\section{2. $P$ and the real numbers $R$}

We start this section by looking at some cardinal invariants of the real numbers $\mathbf{R}$. Recent research has uncovered rather surprising connections between these invariants and the size of certain subgroups of $\mathbf{P}$.

Definition (1) The additivity of measure, $\operatorname{add}(\mathbf{L})$, is the smallest number of measure-zero subsets of $\mathbf{R}$ whose union is not of measure zero.

(2) The additivity of category, add(B), is the smallest number of first category subsets of $\mathbf{R}$ whose union is of second category (not of first category).

(3) The cardinal $\mathbf{d}$, the dominating number, is defined as:

$$
\begin{aligned}
& \min \left\{|D|: D \text { is a subset of } \mathbf{N}^{\mathbf{N}}\right. \text { and } \\
& \left.\left(\forall g \in \mathbf{N}^{\mathbf{N}}\right)(\exists f \in D)(g(n) \leq f(n) \text { for all but finitely many } n)\right\} .
\end{aligned}
$$

(4) The cardinal b, the bounding number, is defined as:

$$
\begin{aligned}
& \min \left\{|B|: \quad B \text { is a subset of } \mathbf{N}^{\mathbf{N}}\right. \text { and } \\
& \left.\left(\forall g \in \mathbf{N}^{\mathbf{N}}\right)(\exists f \in B)(g(n)<f(n) \text { for all but finitely many } n)\right\} .
\end{aligned}
$$

The countable additivity of Lebesgue measure and the Baire category theorem imply that $\operatorname{add}(\mathbf{L})$ and $\operatorname{add}(\mathbf{B})$ are both at least $\omega_{1}$. And they are also at most $2^{\omega}$. It is immediate too that $\omega_{1} \leq \mathbf{b} \leq \mathbf{d} \leq 2^{\omega}$. 
The reason why the dominating and bounding numbers are called invariants of the reals is that the irrationals are homeomorphic to the topological space $\mathbf{N}^{\mathbf{N}}$ when $\mathbf{N}^{\mathbf{N}}$ is given the product topology and $\mathbf{N}$ has the discrete topology.

We shall need one other invariant which is called the pseudointersection number. A family $F$ of subsets of $\mathbf{N}$ has the strong finite intersection property (SFIP) if the intersection of every finite subfamily is an infinite set. For example, the family of cofinite subsets of $\mathbf{N}$ has the SFIP. A set $A$ is almost contained in a set $B$ if $A \backslash B$ is finite.

Definition The cardinal $\mathbf{p}$ is

$\min \{|F|: F$ is a family of subsets of $\mathbf{N}$ such that $F$ has the SFIP but there is no infinite set which is almost contained in every member of $F\}$.

It is an instructive exercise to show that $\omega_{1} \leq \mathbf{p} \leq 2^{\omega}$.

These cardinals are related as in the following picture, part of the Cichon diagram (except for the cardinal $\mathbf{p}$ ). An arrow relation $\kappa \rightarrow \lambda$ means $\kappa \leq \lambda$ :

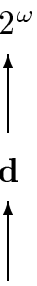

b

Miller [32]

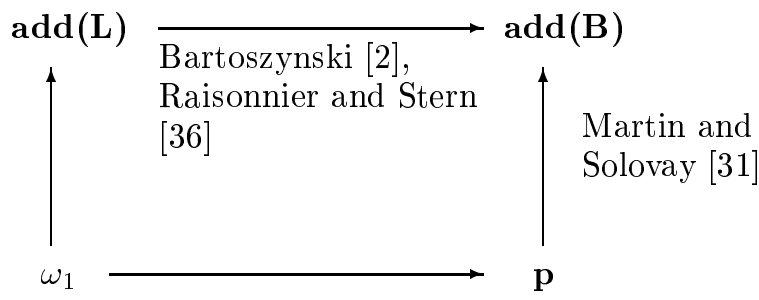


Some of the earliest results on the cardinal invariants of the reals are due to Rothberger [37]. The discovery of Martin's Axiom in 1970 stimulated renewed interest in these and other invariants. A fuller account of the area which has been studied in great depth by mathematicians since the 1970's is available in the articles by van Douwen [13] and Vaughan [44]. More recent work has revealed links between cardinal invariants and quadratic forms: several of these invariants determine how large orthogonal complements there are in a quadratic space. This research (on Gross and strongly Gross spaces) is surveyed in the paper [43]. The bounding number $\mathbf{b}$ also appears in recent work (in functional analysis) on metrizable barrelled spaces [38].

To explain the connection of cardinal invariants with the BaerSpecker group $\mathbf{P}$, we shall introduce one further definition.

Definition (Łoš). A group $G$ is slender if whenever $\phi$ is a homomorphism from $\mathbf{P}$ into $G$, then $\phi\left(e_{n}\right)=0$ for all but finitely many $n$, where $e_{n}$ is the element of $\mathbf{P}$ that has 1 at the $n$-th co-ordinate and 0 everywhere else.

There are several important equivalent characterizations of slender groups which we insert here for the sake of completeness and as an aid to intuition.

Theorem 2.1 (Nunke [33], Heinlein [24], Eda (1982, see [20])) The group $G$ is slender if and only if $G$ does not contain a copy of the rationals $\mathbf{Q}$, the cyclic group of order $p \mathbf{Z}(\mathbf{p})$, the $p$-adic integers $\mathbf{J}_{p}$, or $\mathbf{P}$;

equivalently, every homomorphism $\phi$ from $\mathbf{P}$ into $G$ is continuous, where $G$ and $\mathbf{Z}$ are given the discrete topology and $\mathbf{P}$ the product topology;

equivalently, for any family $\left\{G_{i}: i \in I\right\}$ and homomorphism $\phi$ from $\Pi_{i \in I} G_{i}$ to $G$, there are $\omega_{1}$-complete ultrafilters $D_{1}, \ldots, D_{n}$ on $I$ such that

$\left(\forall g \in \Pi_{i \in I} G_{i}\right)$ (if the support of $g,\{i \in I: g(i) \neq 0\}$,

does not belong to $D_{k}(1 \leq k \leq n)$, then $\left.\phi(g)=0\right)$.

Corollary 2.2 Every $\omega_{1}$-free group which does not contain $\mathbf{P}$ is slender. 
Examples The group $\mathbf{Z}$ is slender; every free group is slender. $\mathbf{P}$ is not slender (Specker [42]). Subgroups and direct sums of slender groups are slender.

Specker's proof that $\mathbf{P}$ is not slender works for many other subgroups of $\mathbf{P}$ which exhibit the Specker phenomenon. But these subgroups all have cardinality $2^{\omega}$.

Definition (Eda [15], Blass [8]) (1) A subgroup $G$ of $\mathbf{P}$ exhibits the Specker phenomenon iff $G$ contains a sequence $\left\{g_{n}: n \in\right.$ $\mathbf{N}\}$ of linearly independent elements such that whenever $\phi$ is a homomorphism from $G$ into $\mathbf{Z}$, then $\phi\left(g_{n}\right)=0$ for all but finitely many $n$.

(2) The Specker-Eda number, se, is defined as:

$$
\min \{|G|: G \leq \mathbf{P} \text { exhibits the Specker phenomenon }\} .
$$

Corollary $2.3 \omega_{1} \leq$ se $\leq 2^{\omega}$.

Theorem 2.4 (Eda [15].) (1) $\mathbf{C H}$ implies $\mathbf{s e}=\omega_{1}$.

(2) Martin's Axiom (MA) implies $\mathbf{s e}=2^{\omega}$.

(3) There is a model of ordinary set theory (ZFC) in which se $<$ $2^{\omega}$.

In 1994, Andreas Blass observed that Eda's proofs establish connections between the Specker-Eda number and some of the cardinal invariants of the real numbers which were defined at the beginning of this section.

Theorem 2.5 (Eda [15], Blass [8]) (1) $\mathbf{p} \leq \mathbf{s e} \leq \mathbf{d}$.

$(2) \operatorname{add}(\mathbf{L}) \leq \mathbf{s e} \leq \mathbf{b}$.

Conjecture 2.6 (Blass [8]) se $=\operatorname{add}(B)$.

\section{3. $P$ and infinitary logic}

From the logical point of view, a group $G$ is a structure $\mathbf{G}=$ $\left(G,+^{G},-{ }^{G}, 0^{G}\right)$ which satisfies the axioms for a group. In this section we use $\mathbf{L}$ to denote the vocabulary of groups, that is, $\mathbf{L}$ contains the constant, unary, and binary function symbols $\mathbf{0},-$, and + to name the zero, inverse, and addition of a group. There are other possible choices for the vocabulary of groups, but for the 
sake of definiteness we shall fix $\mathbf{L}$ as above. It is also a fact that all the theorems of logic presented here are true in much greater generality.

The infinitary language $\mathbf{L}_{\infty \kappa}$ is the smallest class of formulas in the vocabulary $\mathbf{L}$ which is closed under negations, conjunctions of arbitrary length, and strings of quantifiers

$$
\left(\exists x_{1} \exists x_{2} \ldots \exists x_{\alpha} \ldots\right)_{(\alpha<\lambda)}
$$

of length $\lambda$ less than $\kappa$. This infinitary language is more expressive than the first-order language of groups where one is limited to negations, finite conjunctions, and finite strings of quantifiers. While the axioms for a group are first-order, many of the interesting group-theoretic properties cannot be expressed by first-order sentences. For example, the following sentence of $\mathbf{L}_{\infty}$ says that a group is torsion:

$$
(\forall g)(g=0 \text { or } 2 g=0 \text { or } 3 g=0 \text { or } \ldots \text { or } n g=0 \ldots) \text {. }
$$

But the concept of torsion cannot be axiomatized in a first-order language. Infinitary logic can express the concepts of $\kappa$-freeness, $\kappa$-purity, $<\kappa$-generatedness and so on. The paper by Barwise [3] is an excellent introduction to the back and forth methods characteristic of infinitary logic. Other useful references for infinitary logics are the book by Barwise [4] and the article by Dickmann [12]. A typical problem in general infinitary model theory involves determining whether there are infinitarily equivalent nonisomorphic models in various cardinalities, [40].

Definition Two groups $A$ and $B$ are $\mathbf{L}_{\infty \kappa^{-}}$equivalent if and only if for every sentence $\varphi$ in $\mathbf{L}_{\infty \kappa}: \varphi$ is true in $A$ iff $\varphi$ is true in $B$.

So $\mathbf{L}_{\infty \kappa}$-equivalent groups cannot be distinguished by a sentence in the infinitary language $\mathbf{L}_{\infty \kappa}$. There is an algebraic characterization of infinitary equivalence which is very useful and perhaps more familiar.

Theorem 3.1 (Karp [27], Benda [6], Calais [9]) Two groups $A$ and $B$ are $\mathbf{L}_{\infty \kappa}$-equivalent if and only if there is a $\kappa$-extendible system of partial isomorphisms from $A$ to $B$. 
A $\kappa$-extendible system of partial isomorphisms from $A$ to $B$ is a family $F$ of isomorphisms between subgroups of $A$ and $B$ which has the $\kappa$-back-and-forth property: if $\phi \in F$ is an isomorphism from $M_{1} \leq A$ onto $N_{1} \leq B$ and $X$ (respectively $Y$ ) is a subset of $A$ (respectively $B$ ) of cardinality less than $\kappa$, then there exists $\psi \in F$ from $M_{2}$ onto $N_{2}$ such that $M_{1} \leq M_{2} \leq A, N_{1} \leq N_{2} \leq B$, $\psi$ extends $\phi$ and $X(Y)$ is a subset of $M_{2}\left(N_{2}\right)$.

Using this algebraic concept, it is easy to check for example that any two uncountable free groups are $\mathbf{L}_{\infty \omega}$-equivalent. $\kappa^{-}$ extendible systems are a natural generalization of Cantor's technique for showing that there is (up to isomorphism) exactly one unbounded dense countable linear order, namely the linearly ordered set of the rational numbers [3]. Indeed, for countable groups, infinitary equivalence and isomorphism are synonymous:

Theorem 3.2 (Scott [39]) If $A$ and $B$ are countable $\mathbf{L}_{\infty \omega^{-}}$ equivalent groups, then $A$ and $B$ are isomorphic.

The infinitary model theory of abelian groups was intensively studied in the 1970's by Barwise, Eklof, Fischer, Gregory, Kueker, and Mekler (see [5, 16, 17, 23, 28] for example). One of the first important results is due to Eklof, who succeeded in determining which groups are infinitarily equivalent to free groups.

Definition A subgroup $A$ of $G$ is $\kappa$-pure if for every subgroup $B$ such that $A \leq B \leq G$ and $B / A$ is $<\kappa$-generated (i.e. generated by fewer than $\kappa$ elements), $A$ is a direct summand of $B$.

Theorem 3.3 (Eklof [16]) A group $G$ is $\mathbf{L}_{\infty \kappa}$-equivalent to a free group if and only if every $<\kappa$-generated subgroup of $G$ is contained in a free, $\kappa$-pure subgroup of $G$.

For the purposes of this exposition, it is sufficient to know the following corollaries.

Corollary 3.4 A group $G$ is $\mathbf{L}_{\infty \omega}$-equivalent to a free group iff every subgroup of $G$ of finite rank is free.

Eklof used this result to deduce a very famous criterion for freeness in countable groups:

Corollary 3.5 (Pontryagin's Criterion [35]) A countable group is free iff every subgroup of finite rank is free. 
Proof: Apply Scott's Theorem 3.2 to Corollary 3.4.

Corollary $\mathbf{3 . 6}$ (Kueker) A group is $\mathbf{L}_{\infty}$-equivalent to a free group iff it is $\omega_{1}$-free.

Now we can return to the Baer-Specker group $\mathbf{P}$ and see what these facts tell us.

Corollary 3.7 (Keisler-Kueker) The Baer-Specker group $\mathbf{P}$ is $\mathbf{L}_{\infty \omega}$-equivalent to a free group. The class of free groups is not definable in $\mathbf{L}_{\infty \omega}$.

Corollary 3.8 (Eklof [16]) The group $\mathbf{P}$ is not $\mathbf{L}_{\infty \omega_{1}}$-equivalent to a free group.

Since free groups are slender, it follows too from Corollary 3.7 that the class of slender groups is not definable in $\mathbf{L}_{\infty \omega}$. It might be tempting to conjecture that $\mathbf{P}$ is not $\mathbf{L}_{\infty \omega_{1}}$-equivalent to a slender group. Another possible suggestion is that $\mathbf{P}$ is not $\mathbf{L}_{\infty \mathbf{s e}}$-equivalent to a slender group. Mekler showed that if $\kappa$ is a strongly compact cardinal, then the class of free groups is definable in $\mathbf{L}_{\infty \kappa}$. This prompts the question whether the class of slender groups is definable in $\mathbf{L}_{\infty \kappa}$ if $\kappa$ is strongly compact. Eklof and Mekler have developed applications of other generalized logics to problems of abelian group theory in the papers [18] and [19].

\section{The lattice of subgroups of $P$}

The broad thrust in this section is to describe some recent research on the complexity of the lattice of subgroups of $\mathbf{P}$. One way to measure this complexity is to study what sorts of groups can be embedded into $\mathbf{P}$. Very generally, the natural questions often have the form whether there are families of maximal possible size of subgroups of $\mathbf{P}$ which are strongly different (non-isomorphic) in some precisely defined sense.

An example of this type of theorem in the context of general abelian groups is the following.

Theorem 4.1 (Eklof, Mekler and Shelah [21]) Under various settheoretic hypotheses, there exist families of maximal possible size of almost free abelian groups which are pairwise almost disjoint (the intersection of any pair contains no non-free subgroup). 
There is an inverse correlation between the size of the family and the strong difference of its members. If one considers families of pure subgroups of $\mathbf{P}$ and takes the notion of strong difference to mean that the only homomorphisms between any pair are those of finite rank, then it is possible to prove the existence of a strongly different family of maximal size.

Theorem 4.2 (Corner and Goldsmith [10]) Let $\mathbf{D}$ be the subgroup of $\mathbf{P}$ containing $\mathbf{S}$ such that $\mathbf{D} / \mathbf{S}$ is the divisible part of $\mathbf{P} / \mathbf{S}$. Let $c=2^{\omega}$. There exists a family $\mathbf{C}$ consisting of $2^{c}$ pure subgroups $G$ of $\mathbf{D}$ with $\mathbf{D} / G$ rank-1 divisible where each $G$ is slender, essentially-indecomposable, essentially-rigid with $\operatorname{End}(G)=\mathbf{Z}+$ $E_{0}(G)$, where $E_{0}(G)$ is the ideal of all endomorphisms of $G$ whose images have finite rank.

A similar type of question is the following: does there exist a family of $2^{\omega_{1}}$ non-isomorphic pure subgroups of $\mathbf{P}$, each of cardinality $\omega_{1}$, such that the intersection of any pair is free? The answer is positive.

Theorem 4.3 (Shelah and Kolman [41]) There exists a family $\left\{G_{\alpha}: \alpha<2^{\omega_{1}}\right\}$ of pure subgroups of $\mathbf{P}$ such that

(1) each $G_{\alpha}$ has cardinality $\omega_{1}$;

(2) if $\alpha \neq \beta$, then $G_{\alpha} \cap G_{\beta}$ is free.

The question whether certain classes of group can be embedded in $\mathbf{P}$ sometimes leads to independence results. Recall that a group $G$ is $\omega_{1}$-separable if every countable subset of $G$ is contained in a free direct summand of $G$.

Theorem 4.4 (Dugas and Irwin [14]) Embeddability of $\omega_{1}-$ separable groups of cardinality $\omega_{1}$ in $\mathbf{P}$ is independent of $Z F C$.

Reflexive subgroups of $\mathbf{P}$ have also been studied in some depth. The following result was known for many years under the additional assumption of the Continuum Hypothesis.

Theorem 4.5 (Ohta [34]) There exists a non-reflexive dual subgroup of $\mathbf{P}$.

The variety displayed in this selection of results on the BaerSpecker group $\mathbf{P}$ illustrates how this easily definable abelian group is a source of interesting research problems whose solutions often 
reveal unexpected connections with problems in other domains of mathematics.

\section{References}

[1] R. Baer, Abelian groups without elements of finite order, Duke Math. J. 3 (1937), 68-122.

[2] T. Bartoszynski, Additivity of measure implies additivity of category, Trans. Amer. Math. Soc. 281 (1984), 209-213.

[3] J. Barwise, Back and forth through infinitary logic, pp.5-33 in: M. Morley (ed.), Studies in Model Theory, 1975.

[4] J. Barwise, Admissible Sets and Structures. Springer-Verlag: Berlin, 1975.

[5] J. Barwise and P. C. Eklof, Infinitary properties of abelian torsion groups, Ann. Math. Logic 2 (1970), 25-68.

[6] M. Benda, Reduced products and non-standard logics, J. Sym. Logic 34 (1969), 424-436.

[7] S. Ben-David, On Shelah's compactness of cardinals, Israel J. Math. 31 (1978), 34-56.

[8] A. Blass, Cardinal characteristics and the product of countably many infinite cyclic groups, J. Algebra 169 (1994), 512-540.

[9] J. P. Calais, La méthode de Fraïssé dans les langages infinis, C. R. Acad. Sci. Paris 268 (1969), 785-788.

[10] A. L. S. Corner and B. Goldsmith, On endomorphisms and automorphisms of some pure subgroups of the Baer-Specker group, pp.69-78 in: R. Goebel et al. (eds.), Abelian group theory and related topics, Contemp. Math. 171 (1994).

[11] K. J. Devlin, Constructibility. Springer-Verlag: Berlin, 1984.

[12] M. Dickmann, Larger infinitary languages, Chapter IX in: J. Barwise and S. Feferman (eds.), Model-theoretic Logics. Springer-Verlag: Berlin, 1985.

[13] E. van Douwen, The integers and topology, pp.111-167 in: K. Kunen and J. E. Vaughan (eds.), Handbook of Set-theoretic Topology. NorthHolland: Amsterdam, 1984.

[14] M. Dugas and J. Irwin, On pure subgroups of cartesian products of integers, Results in Math. 15 (1989), 35-52. 
[15] K. Eda, A note on subgroups of $\mathbf{Z}^{\mathbf{N}}$, pp.371-374 in: R.Goebel et al. (eds.), Abelian Group Theory. Lecture Notes in Mathematics 1006. Springer-Verlag: Berlin, 1983.

[16] P. C. Eklof, Infinitary equivalence of abelian groups, Fund. Math. 81 (1974), 305-314.

[17] P. C. Eklof and E. R. Fisher, The elementary theory of abelian groups, Ann. Math. Logic 4 (1972), 115-171.

[18] P. C. Eklof and A. H. Mekler, Infinitary stationary logic and abelian groups, Fund. Math. 112 (1981), 1-15.

[19] P. C. Eklof and A. H. Mekler, Categoricity results for $L_{\infty \kappa}$-free algebras, Ann. Pure and Appl. Logic 37 (1988), 81-99.

[20] P. C. Eklof and A. H. Mekler, Almost Free Modules. North-Holland: Amsterdam, 1990.

[21] P. C. Eklof, A. H. Mekler and S. Shelah, Almost disjoint abelian groups, Israel J. Math. 49 (1984), 34-54.

[22] L. Fuchs, Infinite Abelian Groups, Vol. I. Academic Press: New York, 1970.

[23] J. Gregory, Abelian groups infinitarily equivalent to free groups, Notices Amer. Math. Soc 20 (1973), A-500.

[24] G. Heinlein, Vollreflexive Ringe und schlanke Moduln, doctoral dissertation, Univ. Erlangen, 1971.

[25] W. Hodges, In singular cardinality, locally free algebras are free, Algebra Universalis 12 (1981), 205-259.

[26] T. Jech, Set Theory. Academic Press: New York, 1978.

[27] C. Karp, Finite quantifier equivalence, pp.407-412 in: J. Addison et al. (eds.), The Theory of Models. North-Holland: Amsterdam, 1965.

[28] D. W. Kueker, $L_{\infty \omega_{1}}$-elementarily equivalent models of power $\omega_{1}$, pp. 120-131 in: Logic Year 1979-80, Lecture Notes in Mathematics 859. Springer-Verlag: Berlin, 1981.

[29] K. Kunen, Set Theory. North-Holland: Amsterdam, 1980.

[30] M. Magidor and S. Shelah, When does almost free imply free?, J. Amer. Math. Soc. 7 (1994), 769-830.

[31] D. A. Martin and R. M. Solovay, Internal Cohen extensions, Ann. Math. Logic 2 (1970), 143-178.

[32] A. W. Miller, Additivity of measure implies dominating reals, Proc. Amer. Math. Soc. 91 (1984), 111-117. 
[33] R. Nunke, Slender groups, Acta Sci. Math. (Szeged) 23 (1962), 67-73.

[34] H. Ohta, Chains of strongly non-reflexive dual groups of integer-valued continuous functions, Proc. Amer. Math. Soc. 124 (1996), 961-967.

[35] L. S. Pontryagin, The theory of topological commutative groups, Ann. Math. 35 (1934), 361-388.

[36] J. Raisonnier and J. Stern, The strength of measurability hypotheses, Israel J. Math. 50 (1985), 337-349.

[37] F. Rothberger, Eine Aequivalenz zwischen der Kontinuumhypothese und der Existenz der Lusinschen und Sierpinskischen Mengen, Fund. Math. 30 (1938), 215-217.

[38] S. A. Saxon and L. M. Sánchez-Ruiz, Barrelled countable enlargements and the bounding cardinal, J. London Math. Soc. 53 (1996), 158-166.

[39] D. Scott, Logic with denumerably long formulas and finite strings of quantifiers, pp.329-341 in: J. Addison et al. (eds.), The Theory of Models. North-Holland: Amsterdam, 1965.

[40] S. Shelah, Existence of many $L_{\infty \lambda}$-equivalent, non-isomorphic models of $T$ of power $\lambda$, Ann. Pure and Appl. Logic 34 (1987), 291-310.

[41] S. Shelah and O. Kolman, Almost disjoint pure subgroups of the BaerSpecker group, submitted.

[42] E. Specker, Additive Gruppen von Folgen ganzer Zahlen, Portugaliae Math. 9 (1950), 131-140.

[43] O. Spinas, Cardinal invariants and quadratic forms, pp.563-581 in: H. Judah (ed.), Set Theory of the Reals. Israel Mathematical Conference Proceedings (Gelbart Research Institute, Bar-Ilan University), 1993.

[44] J. E. Vaughan, Small uncountable cardinals and topology, pp.195-218 in: J. van Mill and G. M. Reed (eds.), Open Problems in Topology. North-Holland: Amsterdam, 1990.

Eoin Coleman,

King's College London, Strand, London WC2R 2LS, England email: okolman@member.ams.org 